\title{
Osmotic pump implant for chronic infusion of drugs into the inner ear
}

\author{
J. Nadine Brown, Josef M. Miller *, Richard A. Altschuler and Alfred L. Nuttall \\ Kresge Hearing Research Institute, University of Michigan, 1301 East Ann Street, Ann Arbor, MI 48109-05065, USA \\ (Received 6 March 1993; Revision received 15 June 1993; Accepted 16 June 1993)
}

\begin{abstract}
Continuous long-term delivery of experimental drugs to the cochlea of a small animal, such as a young guinea pig, presents several technical problems. A method of placing and securing a cannula-osmotic pump system is described in this paper. Guinea pigs (225-410 g) were unilaterally implanted with an Alzet micro-pump and cannula for delivery of $20 \mathrm{mM}$ tetrodotoxin (TTX) (six animals) or saline (three animals) for three days $(1 \mu \mathrm{l} / \mathrm{h}$ ). Auditory brainstem responses (ABRs) were recorded under light anesthesia on post-implant day 1 and day 3 and compared with pre-implant baseline values. In all six cochleas infused with TTX, most frequencies showed a $30-60 \mathrm{~dB}$ decrease in sensitivity within $24 \mathrm{~h}$. Saline control animals showed little or no change from baseline sensitivity for most frequencies. In three TTX-infused animals, the cannula-pump unit was removed on day 3, and ABRs were followed during recovery. Most frequencies returned to, or near, pre-implant levels after pump removal but recovery times varied. By day 6 , all animals had recovered post-surgical weight loss and showed a gain of $10-40 \mathrm{~g}$. Brains and cochleas were removed and processed for sectioning. Assessment of the cochlear nucleus of non-recovery TTX-treated animals showed a deafness-related flattening of auditory nerve active zones on the treated side.
\end{abstract}

Guinea pig; Tetrodotoxin; Cochlea; Auditory nerve; Synapse flattening

\section{Introduction}

The experimental delivery of drugs directly to the fluids of the inner ear has provided a powerful technique to examine pharmacological mechanisms involved in transduction and homeostatic processes of the inner ear (Mori et al., 1986; Ryan et al., 1987; Nuttall et al., 1977; Ohlsén et al., 1991; Born and Rubel, 1988). In the majority of cases the delivery systems developed have been appropriate for acutc experimentation. However, recently a number of questions have been raised regarding the chronic effects of modification of inner ear fluid chemistry on function of the inner ear. With these questions new techniques have been developed to permit long-term changes in inner ear fluids. Rubel and colleagues have developed an approach that involves embedding drugs in Elvax dises which may be placed in the round window niche and through diffusion permit the slow introduction of drugs through the round window membrane into the perilymph (Pasic and Rubel, 1989). They used this technique to apply tetrodotoxin (TTX) which depressed auditory nerve activity and resulted in deafness-related cell size decrease in cochlear nucleus neurons (Pasic and Rubel, 1989; Sie and Rubel, 1989).

* Corresponding author. Fax: (313) 764-0014.
Both the depression of eighth nerve activity and the decrease in cell size were reversible. An alternative approach has involved cannulation of scala tympani and mounting a microinfusion system on the skull, permitting delivery of small investigator-controlled doses of drug directly into the perilymph (Kingma et al,, 1992). In contrast to the former method in which the drug dosage is continuous and limited to a few days, this method provides more control of timing of delivery, with periodic delivery and intervening recovery periods possible. Neither method, however, is well-suited for continuous long-term application of drugs. We have therefore modified the microinfusion technique, adding the use of the osmotic pump, to allow for chronic delivery for days, weeks, or months.

The purpose of this paper is to report the technique and demonstrate the effectiveness of pump-delivered TTX in depressing auditory nerve responses, as well as the ability of eighth nerve responses to recover after cessation of drug treatment. The osmotic pump system was also used to determine if TTX-induced depression of eighth nerve activity results in deafness-related flattening of the active zones of auditory nerve synapses and if the flattening is reversible. Flattening of active zones in auditory nerve synapses on spherical cells of the anteroventral cochlear nucleus has been reported previously with other methods of producing deafness (Altschuler et al., 1990; Gulley et al., 1978; Miller et al., 1991; Rees et al., 1985). 


\section{Methods}

For cochlear infusion, a fine-tipped cannula was made as described by Kingma et al. (1992). The infusion tip of the cannula was made by stripping the Teflon coating from 36 gauge platinum iridium wire (Cooner Wire Co., Chatsworth, CA). A $1.25 \mathrm{~cm}$ piece of this Teflon tubing $(\sim 0.13 \mathrm{~mm}$ ID $\times 0.18 \mathrm{~mm}$ OD) was inserted in the end of a $6-8 \mathrm{~cm}$ length of polyurethane tubing $(\sim 1.10 \mathrm{~mm}$ OD $\times 0.64 \mathrm{~mm} \mathrm{ID)}$ (Micro-Renathane, Braintree Scientific, Inc., Braintree, MA) and secured with silicon rubber (Dow Corning, MDX 4-4210), leaving 5-6 $\mathrm{mm}$ extending as the fine infusion tip. A small drop of silicone rubber placed $0.5 \mathrm{~mm}$ from the fine tip kept the cannula from being introduced too far into scala tympani and helped seal the infusion hole in the cochlea. The osmotic pump (1003D, $1 \mu \mathrm{l} / \mathrm{h}$, or 2002, $0.5 \mu \mathrm{l} / \mathrm{h}$; Alza Corp., Palo Alto, CA) was placed in a $38^{\circ} \mathrm{C} 0.9 \%$ saline bath for 4 $\mathrm{h}$, which allowed the pump to be operational immediately upon implantation.

Pigmented guinea pigs of both sexes, weighing 225$410 \mathrm{~g}$, were anesthetized with xylazine $(10 \mathrm{mg} / \mathrm{kg}, \mathrm{im})$ and ketamine $(40 \mathrm{mg} / \mathrm{kg}$, im); local infusion of $0.25 \%$ bupivacaine was used as necessary. Core body temperature was maintained at $38 \pm 1^{\circ} \mathrm{C}$ with a heating pad. Under aseptic conditions, the left bulla was exposed postauricularly and a $1.0-2.0 \mathrm{~mm}$ hole drilled through the bone for visualization of the cochlea with an operating microscope (Fig. 1). A 25 gauge needle was used to drill a small $(\sim 0.2 \mathrm{~mm})$ hole in the bony wall of the basal turn slightly lateral to the round window, allowing access to scala tympani. The tip of the pre-tilled cannula was inserted into the hole until the silicone drop was seated against the otic capsule; a small piece of fascia was used to help seal the opening. Clamping the pump end of the cannula prevented leakage into the cochlea during placement. A notch or small hole made with the $25 \mathrm{~g}$ needle in the soft bone lateral to the visualization hole was useful in supporting the fine cannula as it was secured to the bulla with dental acrylic.

The cannula was looped around a small stainless steel screw self-tapped into a hole drilled in the skull (Fig. 1) and secured with methyl methacrylate. A subcutaneous pocket was formed between the scapulae to accommodate the pump. The pump was removed from the saline bath, attached to the cannula and implanted, and the wound was closed with interrupted sutures.

Three groups of three guinea pigs, each of which was unilaterally implanted with a pump, were used in this study. The cannula-pump units of two groups were filled with $20 \mu \mathrm{M}$ TTX (Sigma Chemical Co., St. Louis, $\mathrm{MO}$; $0.9 \%$ saline was used for the third group. ABRs were recorded under light anesthesia at nine frequencies $(2-40 \mathrm{kHz})$ in each animal a few days before implantation (baseline) and on post-implant day 1 and day 3. The infused cochleas of five animals (three TTX and two saline) were removed immediately after the last recording while the pumps were still active (at about $68 \mathrm{~h}$ after implantation). For the second TTX group, the cannula-pump unit was removed under anesthesia after the day $3 \mathrm{ABR}$ recording, and ABRs were recorded again on day 6 , after a three day recovery period. ABRs in one animal had not recovered fully by day 8 , but had recovered by day 20 . One

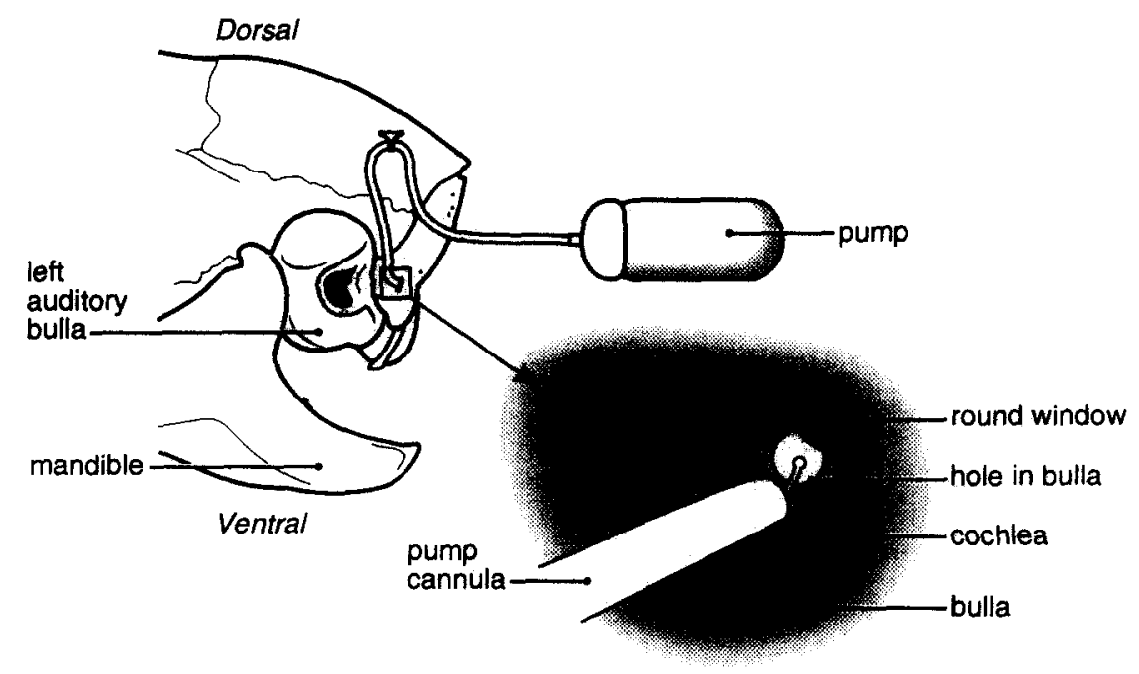

Fig. 1. Diagram of pump and cannula placement in left cochlea of guinea pig. Bone of bulla is indicated with shading. Round window and position of fine cannula tip are visualized through hole drilled in bulla. Cannula is secured with dental cement (not shown) at the bulla and around a small screw in the skull. The pump was implanted subcutaneously between the scapulae. 
animal in this group was implanted with a slower infusion pump $(0.5 \mu \mathrm{l} / \mathrm{h})$ for the three day period.

For tissue fixation, animals were heavily anesthetized with $17.5 \%$ chloral hydrate $(0.2 \mathrm{ml} / 100 \mathrm{~g})$ and perfused through the heart with $0.1 \mathrm{M}$ sodium cacodylate buffer, followed by $3 \%$ glutaraldehyde and $2 \%$ paraformaldehyde in the same buffer. Brains and cochleae were processed into plastic for sectioning. Brains were post-fixed in the same fixative $12-14 \mathrm{~h}$ at $40^{\circ} \mathrm{C}$, rinsed in buffer for $2-3 \mathrm{~h}$, and then sectioned in buffer with a vibrating microtome to yield $100 \mathrm{~mm}$ sections through the cochlear nucleus. The anteroventral cochlear nucleus $(\Lambda \mathrm{VCN})$ was cut from the sections and post-fixed in $1 \%$ osmium tetroxide for $1 \mathrm{~h}$. Sections of AVCN were then dehydrated through a graded series of alcohols and embedded in EmBed 812. Two embedded vibratome sections from comparable regions of AVCN (rostral) from all animals were selected for sectioning and analysis. Thin sections (silver) were cut on a Reichert ultramicrotome and stained with lead citrate and Uranyl acetate. One thin section from each block (two from each animal) was randomly selected for analysis and examined using a JEOL electron microscope. The assessment was made without the observer knowing the treatment condition of the animal. All axo-somatic synapses on all spherical bushy cells in each of the sections were assessed. Primary afferent (auditory nerve) terminals were identified by their vesicles (large, round) and asymmetric post-synaptic density, as well as by size, and their active zones (often more than one per terminal) were identified and assessed as flattened or unflattened. The percent of active zones that were flattened versus unflattened was determined for each section. Cochleae received a local intrascalar perfusion of fixative, followed by 12-14 $\mathrm{h}$ immersion in fixative. They were then decalcified in 3\% EDTA in buffer for 2-3 wccks, dehydrated, and embedded in EmBed 812. Six micron sections were cut, stained with Richardson's stain, and assessed with a Leitz photomicroscope.

This study was reviewed and approved by the University of Michigan Committee on Use and Care of Animals.

\section{Results}

Pre-implant thresholds ( $\mathrm{dB}$ of attention; mean and standard deviation; $N=9$ ) for selected frequencies are: $4 \mathrm{kHz}, 68 \pm 9 ; 12 \mathrm{kHz}, 82 \pm 12 ; 24 \mathrm{kHz}, 76 \pm 9 ; 36 \mathrm{kHz}$, $59 \pm 8$. In all six cochleas infused with TTX, the ABR response at most frequencies showed a $30-60 \mathrm{~dB}$ decrease in sensitivity by day 1 , and this change was maintained or further decreased by $10-40 \mathrm{~dB}$ by day 3 (except in the one animal implanted with the slower infusion pump). Day 3 mean thresholds for TTX ani-

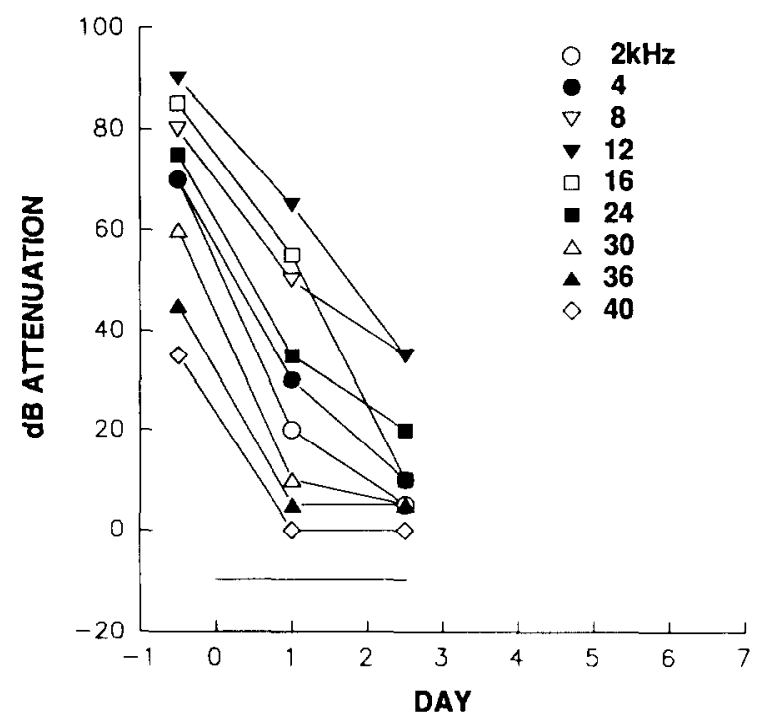

Fig. 2. Effect of TTX cochlear infusion $(20 \mu \mathrm{M}, 1 \mu \mathrm{l} / \mathrm{h})$ in a representative guinea pig. Pump implanted on day 0. ABR thresholds show a $35-75 \mathrm{~dB}$ decrease in sensitivity by post-implant day 2.5 .

mals $(N=5)$ are: $4 \mathrm{kHz}, 11 \pm 10 ; 12 \mathrm{kHz}, 34 \pm 11 ; 24$ $\mathrm{kHz}, 27 \pm 13 ; 36 \mathrm{kHz}, 8 \pm 3$. Typically, suppressed ABRs could be recorded for a wide number of frequencies (Fig. 2).

In the recovery group, most frequencies returned to or near pre-implant levels after the cannula-pump removal, but complete recovery times varied. Response and recovery of one animal is shown in Fig. 3. Pre-implant and final recovery thresholds for the second recovery animal are: $4 \mathrm{kHz} 75,75 ; 12 \mathrm{kHz} 85,60 ; 24$ $\mathrm{kHz} 75,90 ; 36 \mathrm{kHz} 60,80$. In the third animal, implanted with the slower pump, sensitivity loss ranged

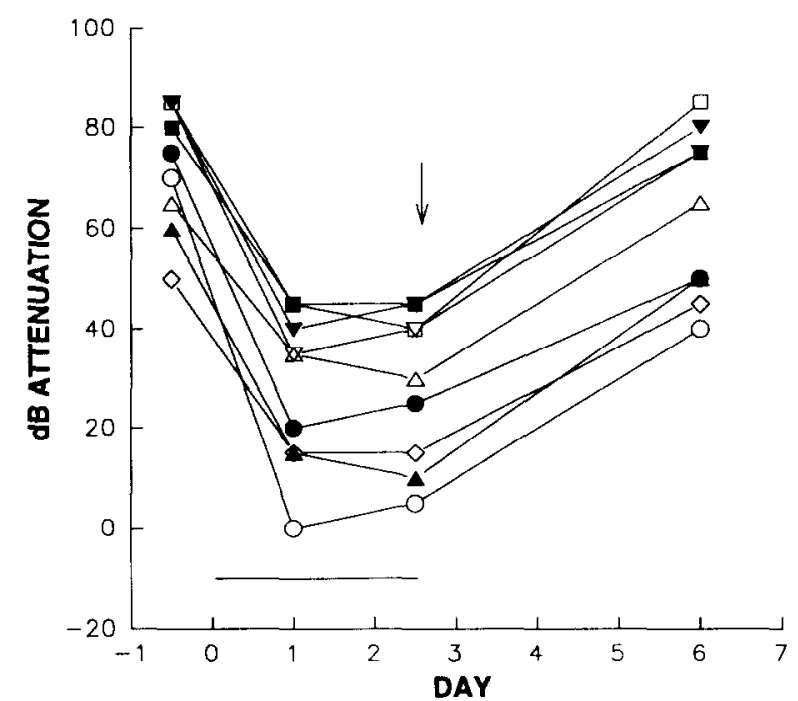

Fig. 3. Recovery from TTX cochlear infusion $(20 \mu \mathrm{M}, 1 \mu \mathrm{l} / \mathrm{h})$ in one guinea pig. Pump implanted on day 0 . The day 1 post-implant sensitivity loss of $30-70 \mathrm{~dB}$ is maintained through day 3. Pump and cannula removed on day 3 (arrow). Day 6 ABR threshold recording shows complete recovery for most frequencies. 
from $10-40 \mathrm{~dB}$ on day 1 . By day 3 , all but one frequency had recovered to within $15 \mathrm{~dB}$ of baseline values. When the cannula-pump unit was exposed for removal, the cannula was found to be dislodged from the bulla. All frequencies were within $15 \mathrm{~dB}$ of baseline by day 7 . TTX and recovery thresholds from this animal are not included in the mean thresholds above.

On day 3 , sensitivity changes in two of the saline controls were within $10 \mathrm{~dB}$ of pre-implant levels at all but the highest tested frequencies (36 and $40 \mathrm{kHz}$ ). Pre-implant thresholds for these two saline animals are: $4 \mathrm{kHz} 70,75 ; 12 \mathrm{kHz} 85,80 ; 24 \mathrm{kHz} 85,80 ; 36 \mathrm{kHz}$ 65,50 . Day 3 thresholds are: $4 \mathrm{kHz} 70,70 ; 12 \mathrm{kHz} 85$. $85 ; 24 \mathrm{kHz} 85,85 ; 36 \mathrm{kHz} 40,65$. In the third control, the tympanic membrane was ruptured during cannula placement, resulting in a $20-60 \mathrm{~dB}$ loss of sensitivity by day 3 . When assessed on day 16 , sensitivity was within $20 \mathrm{~dB}$ of baseline for six frequencies.

Upon recovery from anesthesia for implantation, all TTX animals exhibited moderate to severe twisting of the head and a tendency to circle, which usually diminished in 6-24 $\mathrm{h}$ and thereafter did not significantly
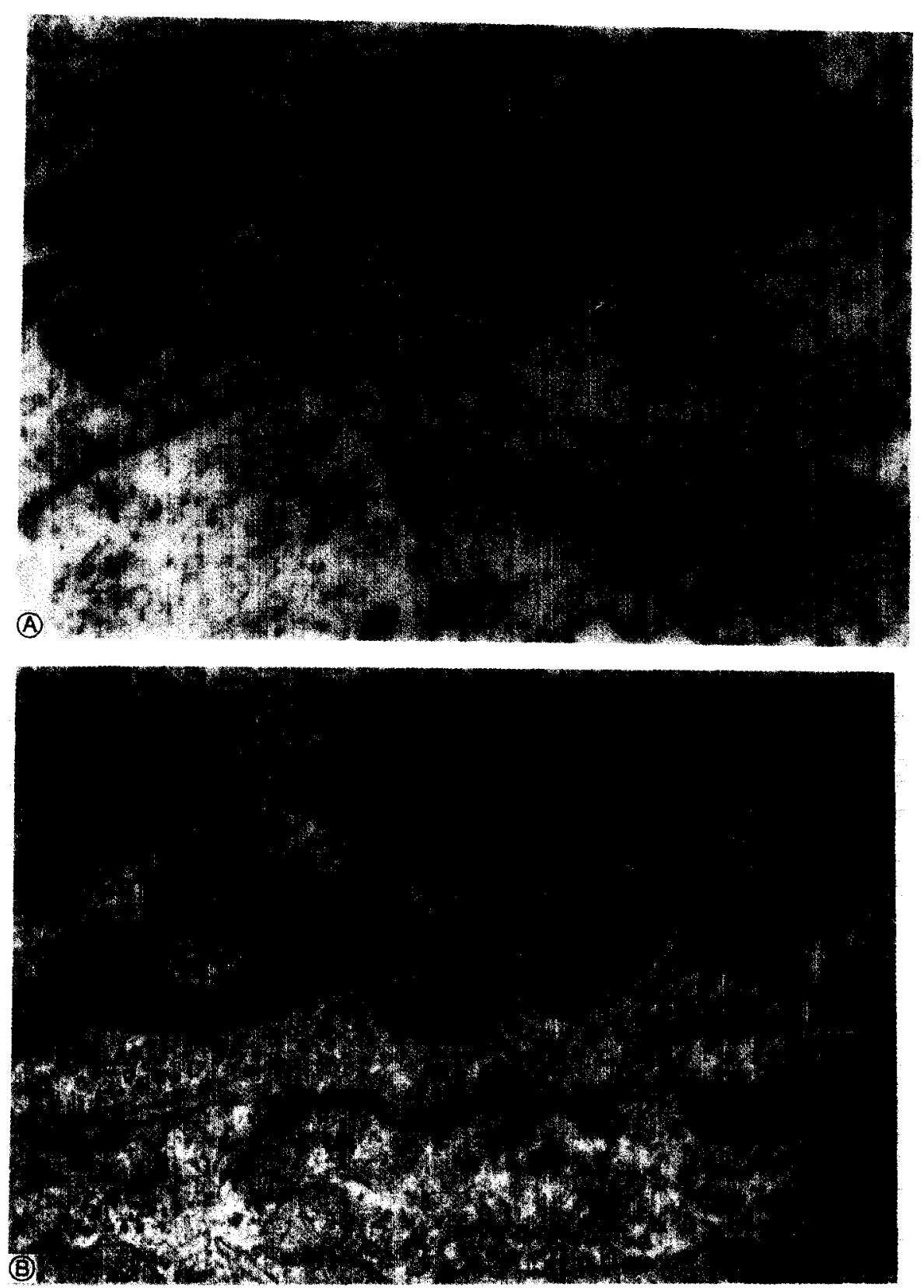

Fig. 4. (A) Flattening of active zones (arrows) was observed in 50-75\% of auditory nerve synapses on spherical bushy cells on the treated side of non-recovery TTX-treated animals. Bar $500 \mathrm{~nm}$. (B) Normal characteristic pre-synaptic invagination and post-synaptic evagination (arrows) was observed in over $90 \%$ of auditory nerve synapses on spherical bushy cells in saline-treated and post-recovery animals. Bar $500 \mathrm{~nm}$. 
affect ability to eat. By day 6 , the three recovery animals had regained post-surgical weight loss and showed a gain of $10-40 \mathrm{~g}$. Little or no post-operative head twist was observed in the three controls.

The middle ears were examined visually under the operating microscope when the bullae were removed or when the cannula-pump units were removed. All appeared normal. After the cannula was removed from recovery animals, the cochlea hole was sealed with a small piece of fascia and the bulla hole covered with Gelfoam. The screw-methyl methacrylate skull attachment was secure in all animals when the cannula was removed so it was left in place; no subscquent problems were noted. In only one animal of the nine was the cannula displaced from the bulla before pump explant.

Flattening of auditory nerve synaptic active zones on spherical bushy cells was observed in the cochlear nucleus on the treated side of non-recovery TTXtreated animals (Fig. 4A). This was not observed on the non-treated side, in control animals, or in animals after recovery from TTX (Fig. 4B). No pathology was observed in the cochleae of drug-treated or control animals.

Not all auditory nerve synapses on spherical bushy cells in TTX-treated animals, however, showed flattening of active zones. Twenty-five to fifty percent of active zones were not flattened. This compares to $10-20 \%$ lack of flattening observed after deafening by kanamycin-ethacrynic acid (Altschuler et al., 1990). There did not appear to be any correlation between tonotopic organization and which synapses showed flattening. In saline-treated control animals and in post-recovery animals, over $90 \%$ of auditory nerve synapses on spherical bushy cells had active zones with the normal characteristic pre-synaptic invagination and post-synaptic evagination.

\section{Discussion}

Osmotic pumps have been used extensively as ratecontrolled drug-delivery systems for numerous organs and tissues in laboratory animals. This paper describes a technique for their use in continuous drug infusion into the cochlea and demonstrates that depression and recovery of cochlear function can be reliably produced in chronically implanted guinea pigs, even of small size. With unilateral infusion of TTX over about three days, a loss of sensitivity of up to $60-65 \mathrm{~dB}$ was observed, followed by recovery to or near baseline values. In our preliminary experiments, under less controlled conditions, infusion of $1 \%$ lidocaine produced similar but less dramatic loss of sensitivity, followed by recovery. ABR stability in the two control implant animals indicates that cochlear cannulation and fluid infusion themselves can have little effect on sensitivity. Lack of full recovery at the highest frequencies in the controls may have been due to local mechanical damage from the implant procedure. However, greater measurement variability is observed at high frequencies, which may also account for some or all of the recorded differences from pre-implant $A B R$ values.

Variations in delivery rate and drug volume can be achieved by use of the various pump models and by varying drug concentration. A sequential discharge of one or more drugs can also be achieved by pre-loading the cannula with alternating immiscible drug or control components (Lynch et al., 1980). Securing the cannula at the top of the head stabilized the distal portion of the cannula, allowing pump replacement if desired, e.g., in studies requiring a prolonged infusion period. Thus, cochlear cannulation with these osmotic pumps as drug source is a technique with many potential clinical and experimental applications.

In the present study, TTX administration produced a flat loss of sensitivity within $24 \mathrm{~h}$, indicating drug perfusion over a fairly large area of the cochlea and/or modiolus, blocking post-ganglionic activity.

Auditory nerve degeneration is seen after inner hair cell loss of varying causes, including aminoglycoside treatment, noise-exposure, and hereditary deafness (Zappia and Altschuler, 1989; Jyung et al., 1989; Leake et al., 1989; Miller et al., 1991; Webster and Webster, 1981). Auditory nerve degeneration in guinea pig reaches $50 \%$ at two months after inner hair cell loss (Jyung et al., 1989; Webster and Webster, 1981) but may take considerably longer in other species, including cat, dog, and human. This raises the question of whether changes in auditory nerve synapses are a result of deafness, i.e., lack of activity in the nerve, or a result of the first stages of degeneration. Our results show that flattening of synapses can result from a reversible cessation of auditory nerve activity, suggesting that it is a deafness-related rather than degeneration-related change.

This study demonstrates that chronic intracochlear delivery of experimental drugs can be achieved with a cannula-osmotic pump system in guinea pigs, even of small size. Such a pump system limits drug distribution to the target tissue, minimizing systemic side effects while permitting precise control of delivery rate, volume, concentration and duration.

\section{Acknowledgments}

Authors gratefully acknowledge Kelly Gizowski for help in preparing the manuscript and Timothy Parrett for help in data collection. This work was supported by NIH grants P01-DC00274, 5RO1 DC00105 and R01DC00383. 


\section{References}

Altschuler, R.A., Horn, J.W., Plattner, E.A. and Miller, I.M. (1990) Prevention of deafness-related changes by cochlear prosthetic stimulation. Abstr. Soc. Neurosci. 20th Ann. Meet. p. 763.

Born, D.E. and Rubel, E.W (1988) Afferent influences on brain stem auditory nuclei of the chicken: Presynaptic action potentials regulate protein synthesis in nucleus magnocellularis neurons. $J$. Neurosci. 8, 901-919.

Gulley, R., Wenthold, R.J. and Neises, G. (1978) Changes in the synapses of spiral ganglion cells in the rostral anteroventral cochlear nucleus of the waltzing guinea pig. Brain Res. 158, $279-294$.

Jyung, R.W., Miller, J.M. and Cannon, S.C. (1989) Evaluation of eighth nerve integrity using the electrically evoked middle latency response. Am. Acad. Otolaryngol. 101, 670-682.

Kingma, G.G., Miller, J.M. and Myers, M.W. (1992) Chronic drug infusion into scala tympani of the guinea pig cochlea. J. Neurosci. Methods 45, 127-134.

leake, P.A., Snyder, R.L., Chambers, P.L., Hradek, G.T., Rebscher, S.J., Bates, G.J. and Marks, D.R. (1989) Consequences of chronic electrical stimulation in an animal model of congenital profound hearing loss. Abstract. Assoc. Res. Otolaryngol. p. 268.

Lynch, H.J., Rivest, R.W. and Wurtman, R.J. (1980) Artificial induction of melatonin rhythms by programmed microinfusion. Neuroendocrinology $31,106-111$.

Miller, J.M., Altschuler, R.A., Niparko, J.K., Hartshorn, D.O., Helfert, R.H. and Moorc, J.K. (1991) Deafness induced changes in the central nervous system and their reversibility and prevention. In: IV International Conference on the Effects of Noise on the Auditory System.
Mori, N., Ohta, K. and Matsunaga, T. (1986) The effect of vasopressin upon the cochlear potentials in the guinea pig. Acta Otolaryngol. 101(3-4), 217-23.

Nuttall, A.L., Marques, D.M. and Lawrence, M. (1977) Effects of perilymphatic perfusion with neomycin on the cochlear micro. phonic potential in the guinea pig. Acta Otolaryngol, 83, 393-400.

Ohlsén, K.A., Baldwin, D.L., Nuttall, A.L. and Miller, J.M. (1991) Influence of topically applied adrenergic agents on cochlear blood flow. Circ. Res. 69, 509-518.

Pasic, T.R. and Rubel, E.W (1989) Rapid changes in cochlear nucleus cell size following blockade of auditory nerve electrical activity in gerbils. J. Comp. Neurol. 283, 474-80.

Rees, S., Guldner, F.H. and Aitkin, L. (1985) Activity dependent plasticity of postsynaptic density structure in the ventral cochlear nucleus of the rat. Brain Res. $325,370-374$.

Ryan, A.F., Schwartz, I.R., Helfert, R.H., Keithley, E. and Wang, Z.X. (1987) Selective retrograde labeling of lateral olivocochlear neurons in the brainstem based on preferential uptake of $3 \mathrm{H}-\mathrm{D}$ aspartic acid in the cochlea. J. Comp. Neurol. 255, 606-16.

Sie, K. and Rubel, E.W (1989) The early effects of eighth nerve blockade on protein synthesis and cell size in the anteroventral cochlear nucleus of the young gerbil. Abstract. Assoc. Res. Otolaryngol. p. 9.

Webster, M. and Webster, D.B. (1981) Spiral ganglion neuron loss following organ of Corti loss: A quantitative study. Brain Res. $212,17-30$.

Zappia, J.J, and Altschuler, R.A. (1989) Evaluation of the effect of ototopical neomycin on spiral ganglion cell density in the guinea pig. Hear. Res. 40, 29-38. 